\title{
STRUCTURAL PROPERTIES FOR TWO CLASSES OF COMBINED RANDOM NUMBER GENERATORS
}

\author{
PIERRE L'ECUYER AND SHU TEZUKA
}

\begin{abstract}
We analyze a class of combined random number generators recently proposed by L'Ecuyer, which combines a set of linear congruential generators (LCG's) with distinct prime moduli. We show that the geometrical behavior of the vectors of points produced by the combined generator can be approximated by the lattice structure of an associated LCG, whose modulus is the product of the moduli of the individual components. The approximation is good if these individual moduli are near each other and if the dimension of the vectors is large enough. The associated LCG is also exactly equivalent to a slightly different combined generator of the form suggested by Wichmann and Hill. We give illustrations, for which we examine the approximation error and assess the quality of the lattice structure of the associated LCG.
\end{abstract}

\section{APPROXIMATING A COMBINED GENERATOR By AN LCG}

Consider $J$ linear congruential generators (LCG's), $J \geq 2$, such that for $j=1, \ldots, J$, generator $j$ has modulus $m_{j}$ and multiplier $a_{j}$. Suppose that the $m_{j}$ 's are all distinct primes and that each LCG has maximal period $m_{j}-1$ $\left(a_{j}\right.$ is a primitive element modulo $\left.m_{j}\right)$. Let $s_{j i}$ denote the state of generator $j$ at step $i$, that is,

$$
s_{j i}:=a_{j} s_{j, i-1} \bmod m_{j} .
$$

Let $\delta_{1}, \ldots, \delta_{J}$ be arbitrary nonzero integers. Define the two combined generators

$$
Z_{i}=\left(\sum_{j=1}^{J} \delta_{j} s_{j i}\right) \bmod m_{1}, \quad U_{i}=Z_{i} / m_{1}
$$

and

$$
W_{i}=\left(\sum_{j=1}^{J} \frac{\delta_{j} s_{j i}}{m_{j}}\right) \bmod 1
$$

Received July 31, 1990.

1980 Mathematics Subject Classification (1985 Revision). Primary 65C10.

Key words and phrases. Random number generation, lattice structure, combined generators, Chinese Remainder Theorem. 
The former is suggested in [5] (with $\delta_{j}=(-1)^{j-1}$, for ease of implementation), while the latter generalizes Wichmann and Hill [11]. Let

$$
\begin{gathered}
n_{j}=\left(\frac{m}{m_{j}}\right)^{m_{j}-2} \bmod m_{j} \text { for } j=1, \ldots, J, \\
m=\prod_{j=1}^{J} m_{j}, \\
a=\left(\sum_{j=1}^{J} \frac{a_{j} n_{j} m}{m_{j}}\right) \bmod m,
\end{gathered}
$$

and define the LCG (with composite modulus):

$$
Y_{i}:=a Y_{i-1} \bmod m, \quad \tilde{U}_{i}=Y_{i} / m
$$

In Proposition 1 below, we show that the combined generator (3) is equivalent to the LCG (7). This is related to the Chinese Remainder Theorem and means that (3) is in fact an implementation of (7) using modular arithmetic [4, §4.3.2]. An alternative approach for computing $a$ is also given in [4, p. 274]. In Proposition 2, we show that if the $m_{j}$ 's are near each other, generator (2) is approximately equivalent to (7) and (3), with some added "noise." We give tight bounds on the noise. This approximation is valid under the assumption that to produce $U(0,1)$ variates, the generator's state is simply divided by the modulus, as in (2) and (7). These results were derived in [9] for the special case $J=2$ and $\delta_{1}=\delta_{2}=1$. Note that $a$ and $m$ do not depend on the $\delta_{j}$ 's. A corollary to Proposition 1 is that the period length of (3) is equal to the Carmichael's function $\lambda(m)$, which in this case is equal to the least common multiple of $m_{1}-1, \ldots, m_{J}-1$.

Proposition 1. If $Y_{0} / m=W_{0}$, then $\widetilde{U}_{i}=Y_{i} / m=W_{i}$ for all $i \geq 0$.

Proof. From the definition of $n_{j}$ and from Fermat's Little Theorem (see, e.g., $[8])$, one has

$$
n_{j}\left(\frac{m}{m_{j}}\right) \bmod m_{j}=\left(\frac{m}{m_{j}}\right)^{m_{j}-1} \bmod m_{j}=1,
$$

so that $n_{j} m / m_{j}=1+K m_{j}$ for some integer $K$ and

$$
n_{j}\left(\frac{m}{m_{j}}\right)^{2} \bmod m=\frac{m}{m_{j}}\left(1+K m_{j}\right) \bmod m=\frac{m}{m_{j}} .
$$


From this, and since $\left(m / m_{k}\right)\left(m / m_{j}\right) \bmod m=0$ for $k \neq j$, one gets

$$
\begin{aligned}
a m W_{i} \bmod m & =\left(\sum_{k=1}^{J} \frac{a_{k} n_{k} m}{m_{k}}\right)\left(\sum_{j=1}^{J} \frac{m \delta_{j} s_{j i}}{m_{j}}\right) \bmod m \\
& =\left(\sum_{j=1}^{J} n_{j}\left(\frac{m}{m_{j}}\right)^{2} a_{j} \delta_{j} s_{j i}\right) \bmod m \\
& =\left(\sum_{j=1}^{J}\left(\frac{m}{m_{j}}\right) \delta_{j} a_{j} s_{j i}\right) \bmod m \\
& =\left(\sum_{j=1}^{J}\left(\frac{m}{m_{j}}\right) \delta_{j}\left(a_{j} s_{j i} \bmod m_{j}\right)\right) \bmod m=m W_{i+1} .
\end{aligned}
$$

Therefore, $m W_{i}$ satisfies the recursion (7), the same as $Y_{i}$.

Corollary 1. The period of $(3)$ (and (7)) is always equal to $\lambda(m)$, provided that for all $j$, we have $\left(\delta_{j} \bmod m_{j}\right) \neq 0$ and $\left(s_{j 0} \bmod m_{j}\right) \neq 0$.

Proof. It suffices to show that $Y_{0}=m W_{0}$ is prime to $m$ and that $a$ is a primitive element modulo $m$, and the result will follow from Carmichael's Theorem [4, §3.2.1.2]. Under the assumption of the corollary, since $m_{j}$ is prime, $\delta_{j} s_{j 0} m / m_{j}$ is prime to $m_{j}$, and $m W_{0}=\sum_{k=1}^{J} \delta_{k} s_{k 0} m / m_{k}$ too, because in this sum, all terms with indices $k \neq j$ are multiples of $m_{j}$. Since this holds for all $j, m W_{0}$ is prime to all prime factors of $m$, that is, prime to $m$. Saying that $a$ is a primitive element modulo $m$ means that there is no positive integer $k$ smaller than $\lambda(m)$ such that $a^{k} \bmod m=1$. If such a $k$ exists, then $a_{j}^{k} \bmod m_{j}=a^{k} \bmod m_{j}=1$ because $a \bmod m_{j}=\left(a_{j} n_{j} m / m_{j}\right) \bmod m_{j}=a_{j}$ from (8). But since $a_{j}$ is a primitive element modulo $m_{j}, k$ must be a multiple of $\lambda\left(m_{j}\right)=m_{j}-1$. Since this holds for all $j, k$ must be a multiple of $\lambda(m)$.

Here, the period of (7) is much smaller than $m-1$ (for $J \geq 2$ ) because the set of states $\{1, \ldots, m-1\}$ is partitioned into subcycles. Of course, it is possible to recover the full period by juxtaposing or interleaving the subcycles. But this complicates the implementation and does not appear to be really helpful in practice.

Define

$$
\begin{aligned}
\Psi^{+} & =\left\{j \mid 2 \leq j \leq J \text { and }\left(m_{j}-m_{1}\right) \delta_{j}>0\right\} \\
\Psi^{-} & =\left\{j \mid 2 \leq j \leq J \text { and }\left(m_{j}-m_{1}\right) \delta_{j}<0\right\} \\
\Delta^{+} & =\sum_{j \in \Psi^{+}} \frac{\left(m_{j}-m_{1}\right)\left(m_{j}-1\right) \delta_{j}}{m_{1} m_{j}}+\sum_{j \in \Psi^{-}} \frac{\left(m_{j}-m_{1}\right) \delta_{j}}{m_{1} m_{j}}, \\
\Delta^{-} & =\sum_{j \in \Psi^{+}} \frac{\left(m_{j}-m_{1}\right) \delta_{j}}{m_{1} m_{j}}+\sum_{j \in \Psi^{-}} \frac{\left(m_{j}-m_{1}\right)\left(m_{j}-1\right) \delta_{j}}{m_{1} m_{j}}, \\
\Delta & =\max \left(\left|\Delta^{+}\right|,\left|\Delta^{-}\right|\right) .
\end{aligned}
$$


Proposition 2. If $Y_{0} / m=W_{0}$, then

$$
U_{i}=\left(W_{i}+\varepsilon_{i}\right) \bmod 1 \text {, }
$$

where

$$
\Delta^{-} \leq \varepsilon_{i} \leq \Delta^{+} .
$$

Proof. For some integer $K$, one has

$$
\begin{aligned}
Z_{i} & =\left(\sum_{j=1}^{J}\left[\frac{m}{m_{j}} \frac{m_{1}}{m}+1-\frac{m_{1}}{m_{j}}\right] \delta_{j} s_{j i}\right) \bmod m_{1} \\
& =\left(\frac{m_{1}}{m}\left(Y_{i}+K m\right)+\sum_{j=1}^{J}\left(1-\frac{m_{1}}{m_{j}}\right) \delta_{j} s_{j i}\right) \bmod m_{1} \\
& =\left(\frac{m_{1} Y_{i}}{m}+m_{1} \varepsilon_{i}\right) \bmod m_{1},
\end{aligned}
$$

where

$$
m_{1} \varepsilon_{i}=\sum_{j=1}^{J}\left(1-\frac{m_{1}}{m_{j}}\right) \delta_{j} s_{j i}=\sum_{j=2}^{J} \frac{\left(m_{j}-m_{1}\right) \delta_{j} s_{j i}}{m_{j}} .
$$

Upon division by $m_{1}$, and since $1 \leq s_{i j} \leq m_{j}-1$ for all $j,(9)$ and (10) follow easily.

Note that the bounds on $\varepsilon_{i}$ are tight, since $\varepsilon_{i}=\Delta^{+}\left[\varepsilon_{i}=\Delta^{-}\right]$(respectively) when $s_{j i}=m_{j}-1$ for $j \in \Psi^{+}$[for $j \in \Psi^{-}$] and $s_{j i}=1$ for $j \in \Psi^{-}$ [for $j \in \Psi^{+}$]. For example, let $J=2, \delta_{1}=1, \delta_{2}=-1$, and $m_{1}>m_{2}$. Then, $\Psi^{+}=\{2\}, \Psi^{-}$is empty, $\Delta^{+}=\left(m_{1}-m_{2}\right)\left(m_{2}-1\right) /\left(m_{1} m_{2}\right), \Delta^{-}=$ $\left(m_{1}-m_{2}\right) /\left(m_{1} m_{2}\right)$, and $\varepsilon_{i}=\left(m_{1}-m_{2}\right) s_{2 i} /\left(m_{1} m_{2}\right)$. We have $\varepsilon_{i}=\Delta^{+}$when $s_{2 i}=m_{2}-1$, and $\varepsilon_{i}=\Delta^{-}$when $s_{2 i}=1$.

From now on, assume that $Y_{i} / m=W_{i}$. It is well known $[3,4]$ that all $t$-tuples of successive values $\left\{\tilde{P}_{i}^{t}=\left(\widetilde{U}_{i}, \ldots, \widetilde{U}_{i+t-1}\right)=\left(W_{i}, \ldots, W_{i+t-1}\right) \in[0,1)^{t}\right.$, $i \geq 0\}$ lie on a lattice $\widetilde{L}_{t}$. Different "figures of merit," relative to the geometrical properties of $\widetilde{L}_{t}$, can be computed for "rating" the corresponding LCG. Among them are the Beyer quotient $q_{t} \in(0,1]$ and the distance $d_{t}$ between successive hyperplanes covering the points $[2,4,3]$. It is traditionally accepted that $q_{t}$ should be near one for all values of $t$ up to a certain constant $T$ (or for which $q_{t}$ can be computed). But the generator's quality also depends strongly on the modulus. As argued in [7], a generator with larger modulus (or of higher order), even if it has a smaller $q_{t}$, might be better. A good "bottom of the line" criterion is in fact the distance $d_{t}$ between hyperplanes. Reducing $d_{t}$ in all dimensions $t$ should be considered as an improvement.

Note that for $J \geq 2$, the $t$-tuples $\widetilde{P}_{i}^{t}$ form a strict subset of the lattice points in $[0,1)^{t}$, since generator (7) does not have maximal period ( $m$ is not prime). But if we take all $t$-tuples of successive values produced by all subcycles of the 
generator, then this set of points is $\widetilde{L}_{t} \cap[0,1)^{t}$ for some lattice $\widetilde{L}_{t}$, and this is the lattice that we analyze in this paper.

The points $\left\{P_{i}^{t}=\left(U_{i}, \ldots, U_{i+t-1}\right), i \geq 0\right\}$ do not belong in general to $\tilde{L}_{t}$. But we see from Proposition 2 that the Euclidean distance between $P_{i}^{t}$ and $\widetilde{P}_{i}^{t}$ obeys

$$
\left\|P_{i}^{t}-\widetilde{P}_{i}^{t}\right\| \leq\left(\varepsilon_{i}^{2}+\cdots+\varepsilon_{i+t-1}^{2}\right)^{1 / 2} \leq \Delta \sqrt{t}
$$

(To take into account the mod 1 operation, consider all the $t$-dimensional unit hypercubes with integer vertices. Each one contains a "representative" of $P_{i}^{t}$, whose coordinates are the same as $P_{i}^{t}$, modulo one. Redefine $\left\|P_{i}^{t}-\widetilde{P}_{i}^{t}\right\|$ as the Euclidean distance between $\widetilde{P}_{i}^{t}$ and the nearest representative of $P_{i}^{t}$.) When $\Delta \sqrt{t}$ is much smaller than $d_{t}$, the combined generator has approximately the same hyperplane structure as its associated LCG. To get rid (to some extent) of the lattice structure (at least in smaller dimensions), one should get a larger $\Delta$. This can be achieved by increasing the values of $\left|\delta_{j}\left(m_{j}-m_{1}\right)\right|$. We remark that $\Delta \sqrt{t}$ is just an upper bound. However, for all the examples that we have examined, that bound was always attained (or almost attained) for some $i$.

\section{THE APPROXIMATE LATTICE STRUCTURE FOR SOME EXAMPLES}

Example 1. Let $J=2, m_{1}=101, m_{2}=97, a_{1}=51, a_{2}=58, \delta_{1}=1$, and $\delta_{2}=-1$. Equations (2) and (3) become respectively $Z_{i}=\left(s_{1 i}-s_{2 i}\right) \bmod 101$ and $W_{i}=\left(s_{1 i} / 101-s_{2 i} / 97\right) \bmod 1$, which have period 2400 . One obtains $m=9797, n_{1}=\left(97^{99} \bmod 101\right)=25, n_{2}=\left(101^{95} \bmod 97\right)=73, a=$ $\left(a_{1} n_{1} m_{2}+a_{2} n_{2} m_{1}\right) \bmod m=2677, \Delta^{-} \approx 0.0004$, and $\Delta=\Delta^{+} \approx 0.0392$. The associated LCG is then

$$
Y_{i}=2677 Y_{i-1} \bmod 9797
$$

Pairs of successive values are plotted in Figures 1-4 for the two combined generators and the two individual components. The latter have small periods and coarse lattice structures. The lattice structure of the LCG (12), which corresponds to the $W_{i}$ 's, is also apparent in two dimensions. Although it is certainly not to be recommended, this generator is nevertheless an improvement over each (much smaller) individual component. The plot for the other combination (the $U_{i}$ 's, in Figure 4 ) looks a little better. The lines of Figure 3 are no longer apparent. In fact, the distance between adjacent lines in Figure 3 is 0.0175 , while the bound in $(11)$ is $\Delta \sqrt{2} \approx 0.0554$. The resolution along each axis is smaller in Figure 4 than in Figure 3: all $U_{i}$ 's are multiples of $1 / m_{1}$, while the $W_{i}$ 's are multiples of $1 / m$, which is much smaller. This is why, in Figure 4 , the points lie on easily discernible equidistant vertical lines, and also on equidistant horizontal lines. 


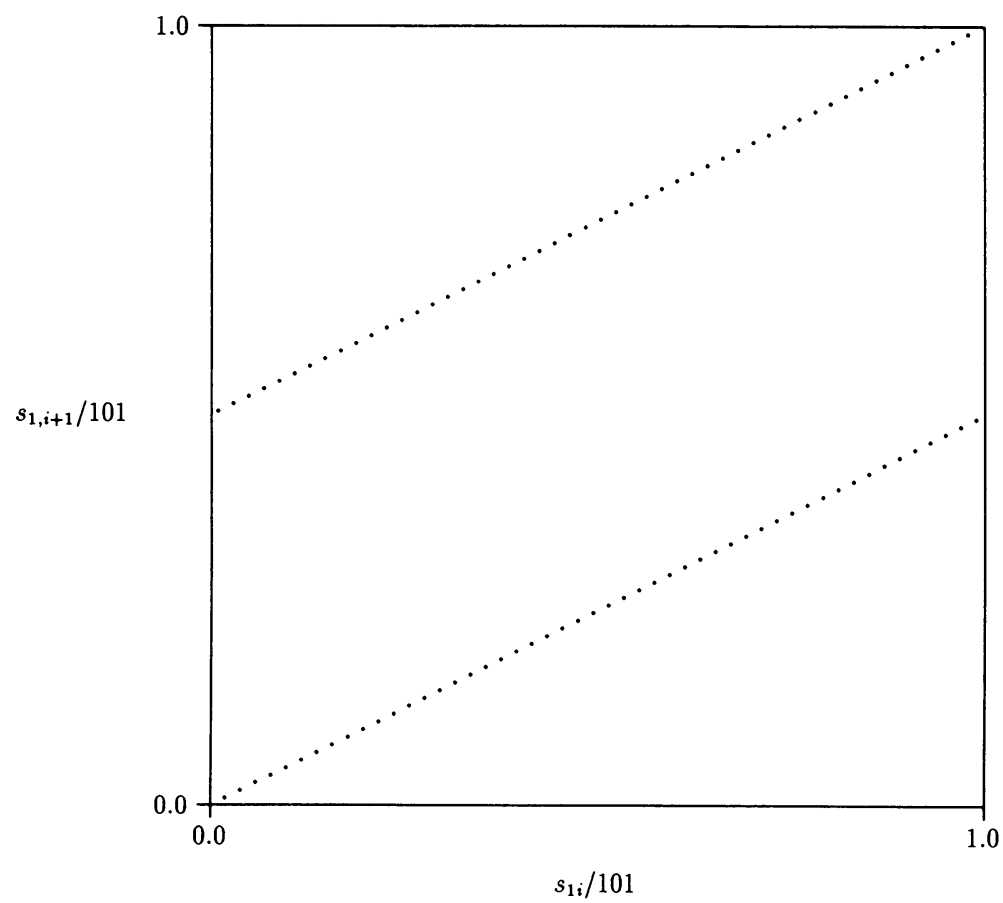

FIGURE 1

All pairs of successive points for the LCG with $m=101$ and $a=51$

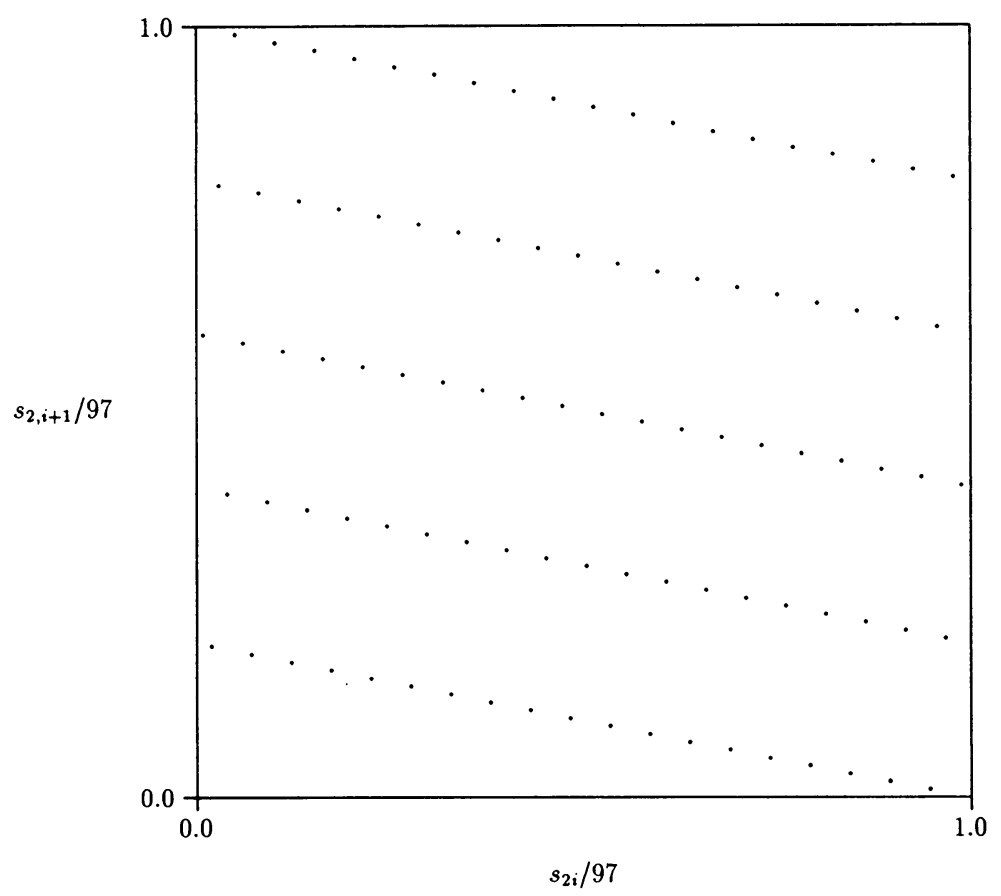

FIGURE 2

All pairs of successive points for the LCG with $m=97$ and $a=58$ 


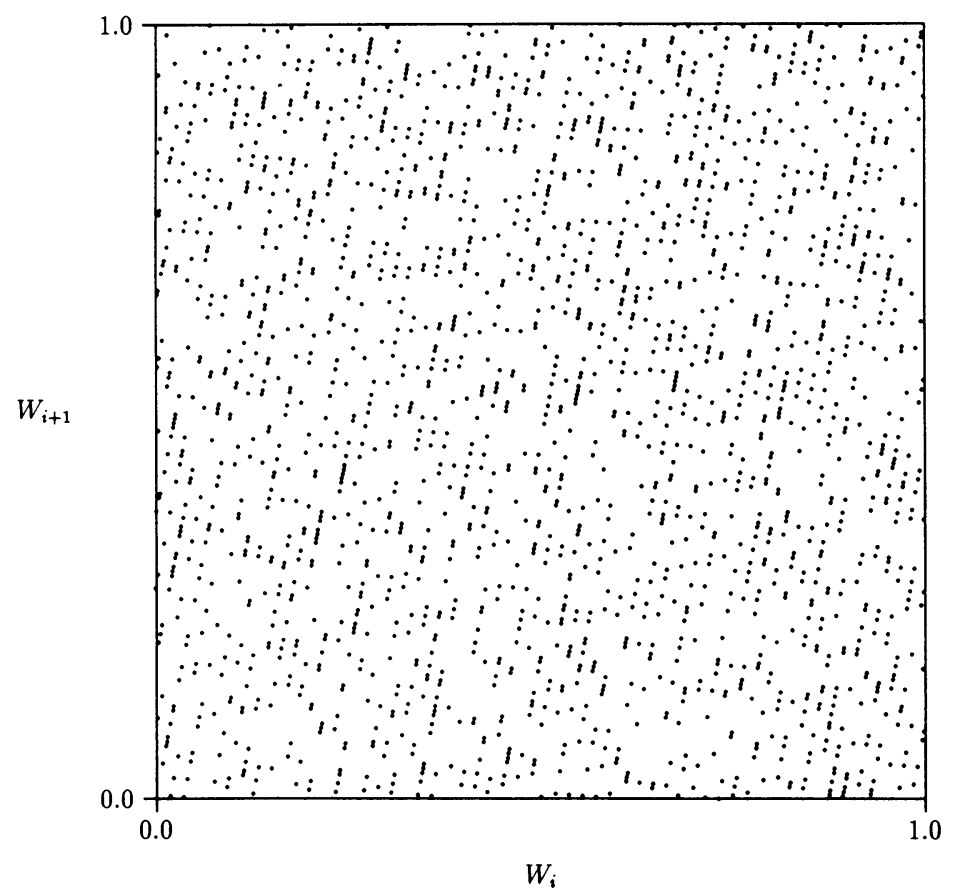

FIGURE 3

Pairs of successive points for the LCG with $m=9797$ and $a=2677$

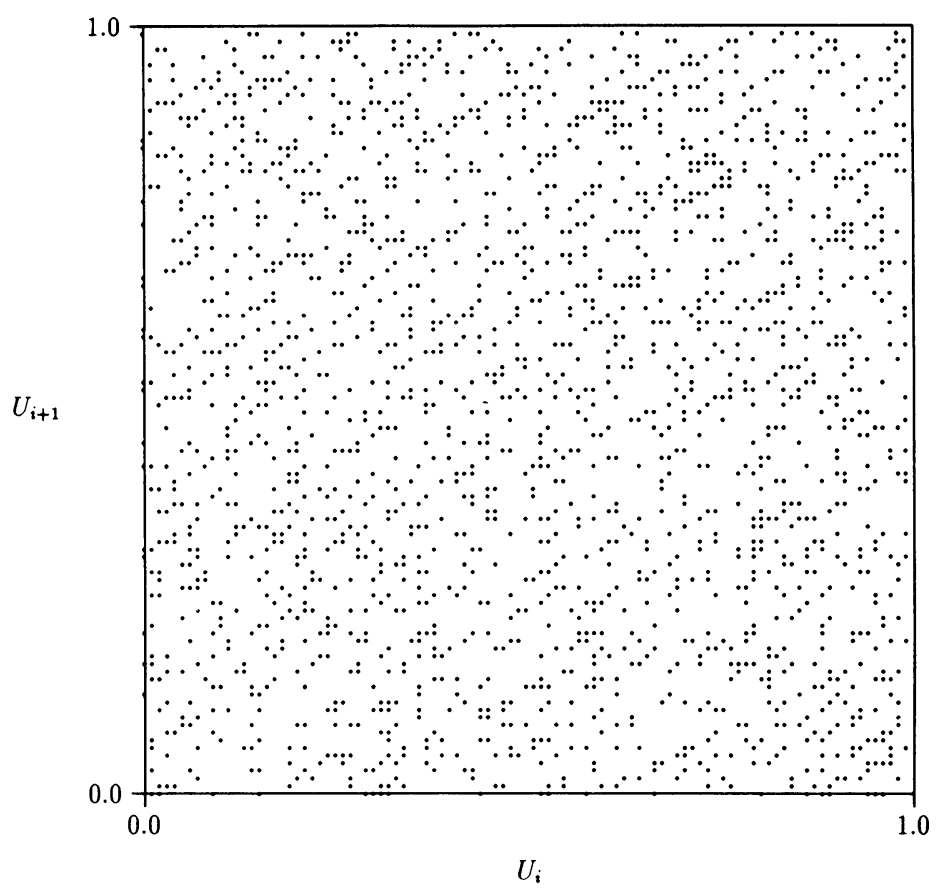

FIGURE 4

Pairs of successive points for the combined generator $U_{i}=$ $\left(\left(s_{1 i}-s_{2 i}\right) \bmod 101\right) / 101$ 
TABLE 1

Results for the LCG's of Example 1

\begin{tabular}{|c|c|c|c|c|c|}
\hline \multirow[b]{2}{*}{$t$} & \multicolumn{3}{|c|}{$(m, a)$} & \multirow{2}{*}{$\begin{array}{r}\left(m_{1}, a_{1}\right) \\
d_{t}\end{array}$} & \multirow{2}{*}{$\begin{array}{r}\left(m_{2}, a_{2}\right) \\
d_{t}\end{array}$} \\
\hline & $q_{t}$ & $d_{t}$ & $\Delta \sqrt{t}$ & & \\
\hline 2 & .3305 & .0175 & .0554 & .447 & .196 \\
\hline 3 & 2479 & .0953 & .0679 & . 447 & .218 \\
\hline 4 & .7597 & .1111 & .0784 & .447 & .377 \\
\hline 5 & .6362 & .1925 & .0876 & .447 & .500 \\
\hline 6 & .8029 & .2182 & .0960 & .447 & .500 \\
\hline 7 & .7395 & .2887 & 1037 & .500 & .500 \\
\hline 8 & .5671 & .4472 & 1109 & .500 & .500 \\
\hline 9 & .5731 & .4472 & .1176 & .500 & .577 \\
\hline 10 & .6400 & .4472 & 1239 & .577 & .577 \\
\hline 11 & .6417 & .4472 & .1300 & .577 & .577 \\
\hline 12 & .7468 & .4472 & .1358 & .577 & .577 \\
\hline
\end{tabular}

In higher dimensions, the distance between hyperplanes typically gets larger, often significantly larger than $\Delta$. In Table 1 , we give the Beyer quotients $q_{t}$, distances $d_{t}$ between hyperplanes, and values of $\Delta \sqrt{t}$ for the LCG (12), for $t \leq 12$. For comparison, we also give the values of $d_{t}$ for the individual LCG components. These quantities were computed using (with some adaptations) the algorithm described in [1].

Example 2. One combined generator which was suggested in [5] has $J=2$, $m_{1}=2147483563, m_{2}=2147483399, a_{1}=40014, a_{2}=40692, \delta_{1}=$ 1 , and $\delta_{2}=-1$. In this case, one has $m=4611685301167870637, n_{1}=$ $1715367968, n_{2}=432115562, a=1968402271571654650, \Delta^{-} \approx 3.5 \times$ $10^{-17}$, and $\Delta=\Delta^{+} \approx 7.637 \times 10^{-8}$. The combined generator (2), as well as its associated LCG $Y_{i}=a Y_{i-1} \bmod m$, have period length of $\left(m_{1}-1\right)\left(m_{2}-1\right) / 2 \approx$ $2.306 \times 10^{18}$. Table 2 gives similar information as Table 1 , for this second example. One can see that in high dimensions, the "noise" $\Delta \sqrt{t}$ becomes very small with respect to the distance between hyperplanes. This was already noticed by Tezuka [9]. On the other hand, the hyperplane structure of the associated LCG is much better than for any of its components, and much better than for any LCG with modulus smaller than $2^{31}$. This is true despite its bad Beyer quotient in dimension 4 . That combined generator has essentially the properties of an LCG with larger modulus $m$ and can be implemented efficiently without getting into the trouble of dealing with large integers (of more than 31 bits). As we will see in Example 4, for the same size, one can also find better combined generators than this one.

Example 3. Wichmann and Hill [11] originally suggested a combination of the form (3), with $J=3, m_{1}=30269, m_{2}=30307, m_{3}=30323, a_{1}=171$, $a_{2}=172, a_{3}=170$, and $\delta_{1}=\delta_{2}=\delta_{3}=1$. This yields $m=27817185604309$, $n_{1}=26478, n_{2}=26070, n_{3}=8037$, and $a=16555425264690$. The equivalence of this generator to an LCG was first pointed out by Zeisel [12]. If 
TABLE 2

The 32-bit combined generator of L'Ecuyer [5]

\begin{tabular}{|c|c|c|c|c|c|}
\hline \multirow[b]{2}{*}{$t$} & \multicolumn{3}{|c|}{$(m, a)$} & \multirow{2}{*}{$\begin{array}{r}\left(m_{1}, a_{1}\right) \\
d_{t}\end{array}$} & \multirow{2}{*}{$\begin{array}{r}\left(m_{2}, a_{2}\right) \\
d_{t}\end{array}$} \\
\hline & $\overline{q_{t}}$ & $d_{t}$ & $\Delta \sqrt{t}$ & & \\
\hline 2 & .5009 & $6.50 \mathrm{E}-10$ & $1.08 \mathrm{E}-7$ & $2.499 \mathrm{E}-5$ & $2.457 \mathrm{E}-5$ \\
\hline 3 & .7016 & $7.002 \mathrm{E}-7$ & 1. $32 \mathrm{E}-7$ & $8.263 E-4$ & $8.441 \mathrm{E}-4$ \\
\hline 4 & .1443 & $4.635 \mathrm{E}-5$ & $1.53 \mathrm{E}-7$ & $4.954 \mathrm{E}-3$ & $4.852 \mathrm{E}-3$ \\
\hline 5 & .5975 & $2.008 \mathrm{E}-4$ & $1.71 \mathrm{E}-7$ & 1. $334 \mathrm{E}-2$ & 1. $240 \mathrm{E}-2$ \\
\hline 6 & .6173 & $8.890 \mathrm{E}-4$ & $1.87 \mathrm{E}-7$ & $2.670 \mathrm{E}-2$ & $2.637 \mathrm{E}-2$ \\
\hline 7 & .6130 & $2.621 \mathrm{E}-3$ & $2.02 \mathrm{E}-7$ & $7.274 \mathrm{E}-2$ & $7.274 \mathrm{E}-2$ \\
\hline 8 & .5737 & $5.782 E-3$ & $2.16 \mathrm{E}-7$ & $7.274 \mathrm{E}-2$ & $7.274 \mathrm{E}-2$ \\
\hline 9 & .5589 & $9.571 \mathrm{E}-3$ & $2.29 E-7$ & $9.806 \mathrm{E}-2$ & $8.737 \mathrm{E}-2$ \\
\hline 10 & .5532 & $1.738 \mathrm{E}-2$ & $2.41 \mathrm{E}-7$ & $1.474 \mathrm{E}-1$ & $1.054 \mathrm{E}-1$ \\
\hline 11 & .6390 & $2.361 \mathrm{E}-2$ & $2.53 \mathrm{E}-7$ & $1.474 \mathrm{E}-1$ & 1. $324 \mathrm{E}-1$ \\
\hline 12 & .6635 & $3.077 \mathrm{E}-2$ & $2.64 \mathrm{E}-7$ & $1.474 \mathrm{E}-1$ & $1.443 \mathrm{E}-1$ \\
\hline
\end{tabular}

one uses equation $(2)$ with these values, one also gets $\Delta^{-} \approx-0.00125$ and $\Delta=$ $\Delta^{+} \approx 0.00178$. L'Ecuyer [5] gave a different one, of the form (2), with $J=3$, $m_{1}=32363, m_{2}=31727, m_{3}=31657, a_{1}=157, a_{2}=146, a_{3}=142$, and $\delta_{1}=-\delta_{2}=\delta_{3}=1$. In that case, one has $m=32504802982957, n_{1}=29617$, $n_{2}=17633, n_{3}=16749, a=30890646900944, \Delta^{+} \approx 0.0196$, and $\Delta=$ $-\Delta^{-} \approx 0.00218$. These generators have respective periods of (approximately) $6.95 \times 10^{12}$ and $8.12 \times 10^{12}$. Tables 3 and 4 give other information on them and on their components. The associated LCG of the second combined generator is bad in dimensions 2 and 6 compared to the first one. But note that even if $q_{2}$ is small, $d_{2}$ is nevertheless smaller in this case than for any standard LCG with modulus $m=2^{31}-1$. Also, the added noise is significantly larger than the distance between hyperplanes, at least up to dimension 12 . The hyperplane structure is lost in the noise. On the other hand, the resolution is only $1 / m_{1}$, which means that all points lie on vertical lines that are 1/32363 apart (and the same horizontally). For this reason, perhaps this generator should not be recommended too strongly for serious applications.

Example 4. We now give an example of a combined generator of roughly the same size as Example 2, whose associated LCG has a lattice structure of slightly better quality, and with much more noise. Incidentally, its two LCG components have bad lattice structures in dimension 3. The first one has $q_{3}=0.0167$ and the second one has $q_{3}=0.1022$. One has $J=2, m_{1}=2147483647$, $m_{2}=2145483479, a_{1}=26756, a_{2}=30318, \delta_{1}=1$, and $\delta_{2}=-1$. In this case, one has $m=4607390686061167913, n_{1}=1317463960, n_{2}=$ $829246600, a=3416908681540390868, \Delta^{-} \approx 4.34 \times 10^{-13}$, and $\Delta=\Delta^{+} \approx$ $9.314 \times 10^{-4}$. The combined generator (2) and its associated LCG (7) have period length of $\left(m_{1}-1\right)\left(m_{2}-1\right) / 2 \approx 2.30 \times 10^{18}$. Table 5 gives further information. Up to dimension 7 , there could be enough noise to mask the hyperplane structure. Also, the smallest Beyer quotient is larger here than for Example 2. 
TABLE 3

The combined generator of Wichmann and Hill [11]

\begin{tabular}{|l|r|r|r|r|r|r|}
\hline \multirow{4}{*}{$t$} & \multicolumn{4}{|c|}{$(m, a)$} & & $\left(m_{1}, a_{1}\right)$ \\
$d_{t}$ & $\begin{array}{r}\left(m_{2}, a_{2}\right) \\
d_{t}\end{array}$ & $\begin{array}{r}\left(m_{3}, a_{3}\right) \\
d_{t}\end{array}$ \\
\cline { 2 - 4 } & $q_{t}$ & $d_{t}$ & $\Delta \sqrt{t}$ & & & \\
2 & .6371 & $2.370 \mathrm{E}-7$ & .0025 & .0058 & .0058 & .0058 \\
3 & .4842 & $4.428 \mathrm{E}-5$ & .0031 & .1562 & .0459 & .0419 \\
4 & .7084 & $5.418 \mathrm{E}-4$ & .0036 & .1562 & .0905 & .1374 \\
5 & .8313 & $2.076 \mathrm{E}-3$ & .0040 & .1562 & .1313 & .1374 \\
6 & .7275 & $6.328 \mathrm{E}-3$ & .0044 & .1690 & .1768 & .2294 \\
7 & .4582 & $1.690 \mathrm{E}-2$ & .0047 & .3536 & .2425 & .2294 \\
8 & .7190 & $2.478 \mathrm{E}-2$ & .0050 & .3536 & .3333 & .3333 \\
9 & .8083 & $2.993 \mathrm{E}-2$ & .0054 & .3536 & .3333 & .3333 \\
10 & .7242 & $4.588 \mathrm{E}-2$ & .0056 & .3536 & .3333 & .3333 \\
11 & .7422 & $5.987 \mathrm{E}-2$ & .0059 & .3536 & .3333 & .3780 \\
12 & .7185 & $7.255 \mathrm{E}-2$ & .0062 & .4472 & .4082 & .3780 \\
\hline
\end{tabular}

TABLE 4

The 16-bit combined generator of L'Ecuyer [5]

$\left.\begin{array}{|l|r|r|r|r|r|r|}\hline \multirow{2}{*}{t} & \multicolumn{3}{|c|}{(m, a)} & \left(m_{1}, a_{1}\right) & \left(m_{2}, a_{2}\right) & \left(m_{3}, a_{3}\right) \\ d_{t} & d_{t}\end{array}\right)$

TABLE 5

A new 32-bit combined generator

\begin{tabular}{|c|c|c|c|c|c|}
\hline \multirow[b]{2}{*}{$t$} & \multicolumn{3}{|c|}{$(m, a)$} & \multirow{2}{*}{$\begin{array}{r}\left(m_{1}, a_{1}\right) \\
d_{t}\end{array}$} & \multirow{2}{*}{$\begin{array}{r}\left(m_{2}, a_{2}\right) \\
d_{t}\end{array}$} \\
\hline & $q_{t}$ & $d_{t}$ & $\Delta \sqrt{t}$ & & \\
\hline 2 & .6934 & $5.54 \mathrm{E}-10$ & 1. $32 \mathrm{E}-3$ & $3.738 E-5$ & $3.298 E-5$ \\
\hline 3 & .7979 & $6.379 E-7$ & $1.61 \mathrm{E}-3$ & $5.138 \mathrm{E}-3$ & $2.986 \mathrm{E}-3$ \\
\hline 4 & .8388 & $2.156 \mathrm{E}-5$ & $1.86 \mathrm{E}-3$ & $5.138 \mathrm{E}-3$ & $5.717 \mathrm{E}-3$ \\
\hline 5 & .9328 & $1.737 \mathrm{E}-4$ & $2.08 \mathrm{E}-3$ & $1.724 \mathrm{E}-2$ & $1.623 \mathrm{E}-2$ \\
\hline 6 & .8074 & $7.731 \mathrm{E}-4$ & $2.28 \mathrm{E}-3$ & $4.046 \mathrm{E}-2$ & $3.400 \mathrm{E}-2$ \\
\hline 7 & .5380 & $2.384 \mathrm{E}-3$ & $2.46 \mathrm{E}-3$ & $4.730 \mathrm{E}-2$ & $5.184 \mathrm{E}-2$ \\
\hline 8 & .7447 & $4.996 \mathrm{E}-3$ & $2.63 \mathrm{E}-3$ & $7.495 \mathrm{E}-2$ & $8.909 E-2$ \\
\hline 9 & .7727 & $9.720 \mathrm{E}-3$ & $2.79 E-3$ & $1.072 \mathrm{E}-1$ & $8.909 E-2$ \\
\hline 10 & .6280 & $1.266 \mathrm{E}-2$ & $2.95 E-3$ & $1.104 \mathrm{E}-1$ & $1.361 E-1$ \\
\hline 11 & .7768 & $1.930 \mathrm{E}-2$ & $3.09 E-3$ & $1.562 \mathrm{E}-1$ & $1.361 \mathrm{E}-1$ \\
\hline 12 & .7795 & $2.859 \mathrm{E}-2$ & $3.22 \mathrm{E}-3$ & $1.562 \mathrm{E}-1$ & $1.474 \mathrm{E}-1$ \\
\hline
\end{tabular}




\section{CONCLUSION}

The combined generators of the forms (3) and (2) are respectively equivalent and approximately equivalent to an LCG. This structural property might appear deceptive at first, because one of the goals of combination was to get rid of the lattice structure of the components. But in fact, they give a stronger theoretical basis to these combination approaches. They show that combination can be viewed as an efficient way of implementing (sometimes with added noise) an LCG with much larger modulus than the largest integer representable on the target computer. If well chosen, that LCG will have much better properties than any of its components. Selecting a combined generator should be based on the properties of its associated LCG rather than on those of its components. After extensive numerical investigations, we found that the quality (in terms of lattice structure) of the associated LCG is essentially unrelated to the quality of its individual components. This means that when searching for good combined generators, searching for individual components with the best lattice structure (as was done in [5]) is essentially useless. When the individual moduli differ enough (with $\delta_{i}= \pm 1$ ), the lattice structure of (7) is usually not recognizable by looking at the points produced by (2) in small dimensions. With appropriate parameters, combination (2) can be used to get rid of the lattice structure up to a given dimension.

An alternative approach, which yields a lattice structure of comparable quality to combination (3) and longer period, is to use a multiple recursive generator of order $J$ (see $[6,7]$ ).

\section{ACKNOWLEDGMENTS}

This work has been supported by NSERC-Canada grant \#A5463 and FCARQuébec grant \#EQ2831. Raymond Couture, Marco Jacques, and François Paradis gave suggestions and helped compute the values for the numerical examples.

\section{BIBLIOGRAPHY}

1. L. Afflerbach and H. Grothe, Calculation of Minkowski-reduced lattice bases, Computing 35 (1985), 269-276.

2. U. Dieter, How to calculate shortest vectors in a lattice, Math. Comp. 29 (1975), 827-833.

3. H. Grothe, Matrixgeneratoren zur Erzeugung gleichverteilter Pseudozufallsvektoren, Dissertation, Tech. Hochschule Darmstadt, Germany, 1988.

4. D. E. Knuth, The art of computer programming: Seminumerical algorithms, Vol. 2, 2nd ed., Addison-Wesley, Reading, MA, 1981.

5. P. L'Ecuyer, Efficient and portable combined random number generators, Comm. ACM 31 (1988), 742-749 and 774. (See also the correspondence in the same journal 32 (1989), 1019-1024.)

6. __ Random numbers for simulation, Comm. ACM 33, 10 (1990), 85-97.

7. P. L'Ecuyer and F. Blouin, Multiple recursive and matrix linear congruential generators (submitted).

8. R. Lidl and H. Niederreiter, Introduction to finite fields and their applications, Cambridge Univ. Press, Cambridge, 1986. 
9. S. Tezuka, Analysis of L'Ecuyer's combined random number generator, Technical report RT-5014, IBM Research, Tokyo Research Laboratory, 1989.

10. S. Tezuka and P. L'Ecuyer, Efficient and portable combined Tausworthe random number generators (submitted).

11. B. A. Wichmann and I. D. Hill, An efficient and portable pseudo-random number generator, Applied Statistics 31 (1982), 188-190. (See also corrections and remarks in the same journal by Wichmann and Hill 33 (1984), 123; McLeod 34 (1985), 198-200.)

12. H. Zeisel, A remark on algorithm AS 183, Applied Statistics 35 (1986), 89.

Département d'IRO, Université de Montréal, C.P. 6128, Succ. A, Montréal H3C 3J7, CANADA

IBM Research, Tokyo Research Laboratory, 5-19, Sanbancho, Chiyodaku, Tokyo 102, JAPAN 